\title{
Comparison of Sentinel and Bactec blood culture
}

\section{systems}

\begin{abstract}
M Stevens, H Patel, A Walters, K Burch, A Jay, N Dowling, C J Mitchell, R A Swann,
\end{abstract} A T Willis, D C Shanson, C A MacDonald

\begin{abstract}
Aims: To evaluate the Sentinel automated blood culture system and to compare its performance with that of Bactec.

Methods: The Sentinel blood culture system was evaluated in three centres. The performance of the system was assessed in comparison with the routine blood culture method used in these centres, the Bactec system.
\end{abstract}

Results: Blood culture sets $(n=2180)$ consisting of Sentinel aerobic and anaerobic, and Bactec aerobic and anaerobic bottles yielded $218(10 \%)$ clinically important isolates. One hundred and fifty five ( $71 \%)$ of the isolates were detected by both systems; 35 (16\%) were detected by Sentinel only; and 28 (13\%) by Bactec only. For the duration of the evaluation, the Sentinel system was deliberately configured so that it was impossible to detect positive results during the first 12 hours. The times to positivity after the first 12 hours were similar. Data gathered during and subsequent to the evaluation have been used by the manufacaturer to refine the algorithm so that positive results can be detected at a minimum of $2 \cdot 25$ hours.

Conclusions: After a period of familiarisation the Sentinel system was considered easy to use. Sentinel is a useful addition to the methods available for the detection of bacteria in blood cultures.

Department of Health Evaluation Unit, Public Health

Laboratory, The Royal

Infirmary, Leicester

LE1 $5 W W$

M Stevens

H Patel

R A Swann

C J Mitchell

Public Health

Laboratory, Luton

A T Willis

K Burch

A Walters

Department of

Medical Microbiology,

Charing Cross and

Westminster Medical

School, London

D C Shanson

A Jay

N Dowling

Medical Devices

Directorate,

Department of Health,

14 Russell Square,

London

C A MacDonald

Correspondence to: M Stevens

Accepted for publication 20 March 1992

Blood cultures represent one of the most important specimens submitted to clinical microbiology laboratories. They are the mainstay of the microbial diagnosis of septicaemia, infective endocarditis, and conditions associated with a clinical presentation of fever of unknown origin. ${ }^{1}$ Many methods have been described and reviewed, ${ }^{2}$ but there remains a need for systems which monitor blood cultures continuously and automatically. Sentinel (Difco Laboratories Ltd, East Molesey, Surrey) is an automated blood culture system that detects bacterial growth by monitoring voltages produced within the bottles containing a blood culture medium mixture.

An evaluation of the system was commissioned by the Medical Devices Directorate of the Department of Health, and carried out at three centres: the Westminster Hospital, London, and the Public Health Laboratories at Luton and Leicester. The system's performance was assessed in comparison with the routine blood culture method used in these centres, the Bactec system. A full report of the evaluation has been produced. ${ }^{3}$

\section{Methods}

SENTINEL SYSTEM

The Sentinel system consists of a base plinth on which are placed between one and five incubation drawers, each holding 80 bottles containing blood and broth culture media (fig 1). A five drawer ( 400 bottle) system measures $112 \mathrm{~cm}$ high, $75 \mathrm{~cm}$ wide, and $75 \mathrm{~cm}$ deep. The bottles contain electrodes and the system detects microbial growth by measuring relative changes in voltage produced in the bottle. The Sentinel bottles contain one gold-plated and one aluminium alloy electrode in the base (fig 2 ). The electrodes are concealed, but on insertion into a Sentinel drawer they pierce a thin membrane and enter the culture medium. This results in the bottle becoming a simple battery, with two dissimilar metals in an electrically conducting fluid. The voltage is generated by the aluminium electrode (anode) slowly dissolving, liberating aluminium ions; the resulting electrons transfer to the gold electrode (cathode) via the drawer circuitry. At the gold electrode, the electrons are transferred to reducable chemical species (electron acceptors) in the medium. In the aerobic system dissolved oxygen is the major electron acceptor, but in the oxygen free anerobic system alternative compounds fulfil this function. When micro-organisms grow, oxygen (in the aerobic bottle) and the alternative electron acceptors (in the anerobic bottle) are reduced

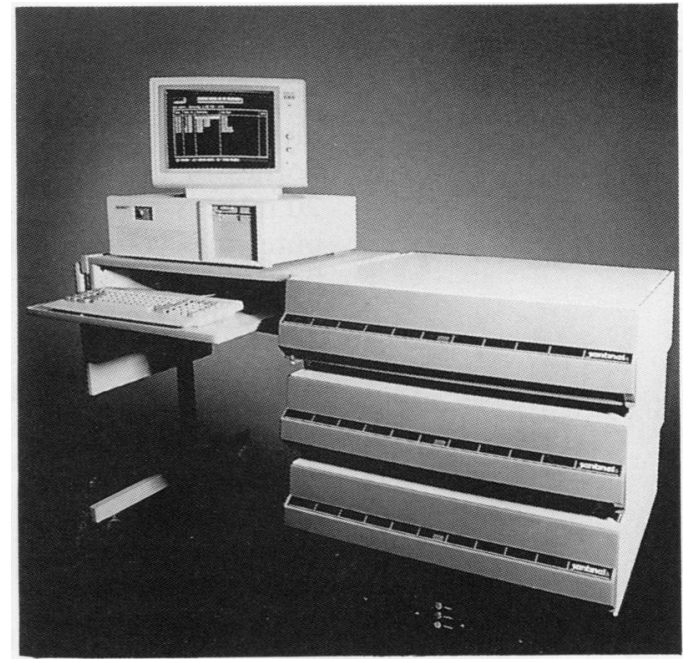

Figure 1 Complete Sentinel three drawer system, with computer. 


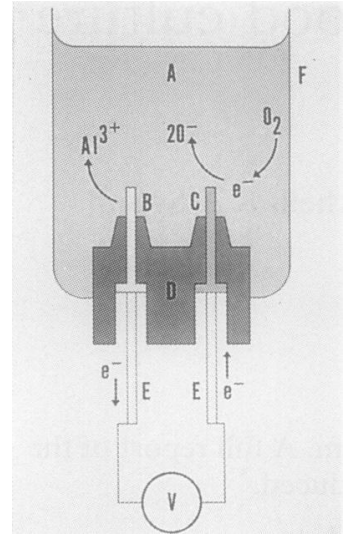

Figure 2 Schematic representations of aerobic Sentinel bottle: $(A)$ medium; (B) aluminium electrode; (C) gold electrode; (D) base rubber bung; (E) electrode connection to drawer; $(F)$ glass bottle; $(V)$ voltage measuring circuit; (e) electron flow. and the voltage between the two electrodes falls.

The drawer electronics detect these voltage changes and an algorithm function within the computer software determines when the voltage has dropped sufficiently to flag the bottle as positive. The Sentinel drawers monitor the voltage between the two electrodes in each bottle at about 14 second intervals. The average value of these readings for each bottle is transmitted to the computer every 15 minutes. Voltage readings less than $40 \mathrm{mv}$ default to zero; that particular 15 minute reading is then excluded from the algorithm. This ensures that removal of bottles does not cause flagging of false positive results. The algorithm has the same format for both aerobic and anerobic bottles, but there are different coefficients used for the two types of bottle. The system checks the absolute voltage (a voltage level test) and any drop in voltage from the previous reading (the fall off test).

The algorithm used during the evaluation allowed a minimum time of 12.5 hours for the detection of a positive result in the aerobic bottle and 3.75 hours in the anaerobic bottle. In the case of the aerobic bottle the longer period for detection was due to incomplete determination of the algorithm coefficients for the test before evaluation began.

EVALUATION METHODS

The evaluation was carried out in three separate laboratories by an agreed protocol. Different Bactec models were used in each of the three participating laboratories. Westminster Hospital used a Bactec 460 (radiometric detection system) using $6 \mathrm{~B}$ and $7 \mathrm{D}$ media, the Public Health Laboratory at Luton used a model NR 730, and the Public Health Laboratory at Leicester used a model NR 660; the latter two systems used infra-red spectroscopy and $6 \mathrm{~A}$ and $7 \mathrm{~A}$ media. The detection thresholds used were the same at all centres: a growth value of $\geq 30$, or, if there was a rise of $\geq 15$ in between readings, the $\delta$ value. Although two different detection systems were used, results are reported as being comparable. $^{45}$

Sets of the Sentinel and Bactec bottles (aerobic and anaerobic in each case) were distributed to hospital wards with instructions that $20 \mathrm{ml}$ of blood should be taken and divided equally between each of the four bottles in the set. In the event that less than $20 \mathrm{ml}$ was taken the user was asked to distribute the volume equally between all four bottles. To determine blood volumes introduced into each type of bottle, $10 \%$ of the total number of Sentinel and Bactec bottles were weighed before despatch to the hospital wards. These bottles were individually coded so that they could be identified and weighed again on return to the laboratory. The weights were converted to volumes by multiplying them by the value of the mean density of human blood $(1.0595 \mathrm{~g} / \mathrm{ml}){ }^{6}$

The time at which bottles were entered into each system and became positive was recorded; this was automatic in the Sentinel system.
All bottles were incubated for a minimum of seven days, longer if there was a clinical requirement, such as in the case of suspected bacterial endocarditis. After completion of the incubation period all bottles were subcultured on to a blood agar plate, for anaerobic incubation, a heated blood agar plate for incubation in an atmosphere of $\mathrm{CO}_{2}$, and a MacConkey agar plate for aerobic incubation. All plates were incubated at $37^{\circ} \mathrm{C}$ for 48 hours.

\section{Results}

A total of 2180 complete sets of blood cultures was received during the evaluation-aerobic and anaerobic bottles for both Bactec and Sentinel. The totals for each of the three centres were Westminster 663, Leicester 922, and Luton 595 specimens. In addition, 304 sets, consisting only of Sentinel and Bactec aerobic bottles were received from paediatric patients.

The 2180 complete sets yielded 218 (10\%) clinically important isolates (table 1). Of these, $35(16 \%)$ were isolated in the Sentinel system only and $28(13 \%)$ in the Bactec system only: $155(71 \%)$ were detected by both systems.

Among the 304 paediatric samples, two were positive; one was Streptococcus pneumoniae, which was detected only in the Bactec aerobic bottle, the other was Haemophilus influenzae, which was detected only in the Sentinel aerobic bottle.

STRAINS ISOLATED ONLY ON TERMINAL SUBCULTURE Ten clinically important strains were isolated only on terminal subculture. Four (Streptococcus pneumoniae, $\beta$ haemolytic streptococcus group C, Staphylococcus aureus and Candida glabrata) were isolated from the Sentinel system. These were not indicated positive by Sentinel but were positive in the Bactec system. Two clinically important isolates, Pseudomonas aeruginosa and Streptococcus sanguis, were isolated on terminal subculture from the Bactec system; these were not indicated positive by Bactec but were positive in the Sentinel system.

In addition, three clinically important isolates (Pseudomonas testosteroni, Gardnerella vaginalis, and Proteus mirabilis) were isolated on terminal subculture from Sentinel bottles, which were not indicated positive by either Sentinel or Bactec and were not isolated from the Bactec bottles. One organism, Pseudomonas aeruginosa, was isolated on terminal subculture from the Bactec system, although it had not been indicated positive by either Bactec or Sentinel; it was not isolated from the Sentinel bottles.

FALSE POSITIVE RESULTS

During the trial, $67(1.5 \%)$ Bactec bottles and $130(3 \%)$ Sentinel bottles indicated positive, but were negative by cultural and microscopical methods.

TIME TO POSITIVITY

The comparison of detection times is shown in table 2. Bactec detected $20 \%$ of the positive 
Table 1 Positive blood cultures yieding clinically important isolates

\begin{tabular}{|c|c|c|c|c|}
\hline Organism & $\begin{array}{l}\text { Both } \\
\text { systems }\end{array}$ & $\begin{array}{l}\text { Sentinel } \\
\text { system } \\
\text { only }\end{array}$ & $\begin{array}{l}\text { Bactec } \\
\text { system } \\
\text { only }\end{array}$ & Total \\
\hline Staphylococcus aureus & 24 & 1 & 5 & 30 \\
\hline Coagulase negative staphylococci & 12 & 2 & & 14 \\
\hline Streptococcus pneumoniae & 16 & 3 & 6 & 25 \\
\hline Streptococus spp & 6 & 2 & 2 & 10 \\
\hline$\beta$ haemolytic streptococci ${ }^{\star}$ & 4 & 1 & 2 & 7 \\
\hline Enterococcus spp & 11 & 6 & 3 & 20 \\
\hline Haemophilis influenzae & 3 & & & 3 \\
\hline Haemophilis parainfluenzae & & & 1 & 1 \\
\hline Escherichia coli & 27 & 7 & 2 & 36 \\
\hline Klebsiella spp & 6 & 2 & 1 & 9 \\
\hline Salmonella spp & 2 & 1 & & 3 \\
\hline Proteus mirabilis & 6 & & & 6 \\
\hline Morganella morganii & 2 & & & 2 \\
\hline Serratia spp & 2 & & 1 & 3 \\
\hline Citrobacter freundii & 2 & 1 & & 3 \\
\hline Enterobacter spp & 8 & & 1 & 9 \\
\hline Acinetobacter calcoaceticus & 1 & & & 1 \\
\hline Pseudomonas aeruginosa & 6 & 4 & 1 & 11 \\
\hline Pseudomonas spp & 9 & & & 9 \\
\hline Aeromonas hydrophila & $i$ & & & 1 \\
\hline Agrobacterium radiobacter & 1 & & & $i$ \\
\hline Clostridium spp & 2 & 3 & & 5 \\
\hline Peptostreptococcus anaerobius & 1 & 1 & & 2 \\
\hline Bacteroides spp & 2 & 1 & 1 & 4 \\
\hline Propionibacterium acnes & & & 1 & 1 \\
\hline Candida spp & 1 & & 1 & 2 \\
\hline Totals & 155 & 35 & 28 & 218 \\
\hline
\end{tabular}

*Includes four group A streptococci and one each of groups B, C, and G.

samples before six hours compared with $0.5 \%$ for Sentinel. This was due to the fact that timings started when samples are "entered" into each system. In the case of the Bactec this was when they were first tested, often after prior incubation.

The average times to positivity for each type of bottle were: Bactec aerobic 22.4 hours; Bactec anaerobic $28 \cdot 7$ hours; Sentinel aerobic $22 \cdot 7$ hours; Sentinel anaerobic $31 \cdot 4$ hours.

\section{AMOUNTS OF BLOOD}

The average amounts of blood introduced into each type of bottle were: Bactec aerobic $3.3 \mathrm{ml}$; bactec anaerobic $3.2 \mathrm{ml}$; Sentinel aerobic $3.9 \mathrm{ml}$; Sentinel anaerobic $3.9 \mathrm{ml}$. The recommended amount is between $3 \mathrm{ml}$ and $5 \mathrm{ml}$ for Bactec bottles and $5 \mathrm{ml}$ into each Sentinel bottle.

A total of $174(8 \%)$ of all sample sets grew organisms that were regarded as not clinically important. Some sets grew such isolates from more than one bottle, so that a total of 278 bottles ( $3 \cdot 2 \%$ of the total of 8720 bottles) grew organisms which were not clinically important. The numbers of each type of bottle which grew non-significant isolates were: Bactec aerobic $88(4 \cdot 0 \%)$, Bactec anaerobic $61(2 \cdot 8 \%)$, Sentinel aerobic $78(3 \cdot 6 \%)$, Sentinel anaerobic 51 $(2 \cdot 3 \%)$.

\section{Discussion}

The results indicated that the Sentinel system was at least as good as the Bactec systems in the rate of detection of organisms.

Sentinel detected more isolates than Bactec but the numbers were small and the difference not significant. The types of organisms detected were diverse, doubtless reflecting the different patient populations tested in a multicentre evaluation. The range of organisms detected was comparable in both systems. The number of complete blood culture sample sets examined (2180) and the significant positive rate (218) over the period of evaluation, exceeded the minimum numbers $(2000$ and 200 respectively) recommended by Illstrup. ${ }^{7}$ Of particular note was the fact that Sentinel detected seven isolates of Escherichia coli which were not detected by Bactec while Bactec in turn detected two strains of $E$ coli which Sentinel did not; $E$ coli was detected in 27 samples by both systems. Bactec detected six isolates of Streptococcus pneumoniae which Sentinel did not; Sentinel detected three isolates which Bactec did not; 16 isolates of $S$ pneumoniae were detected by both systems. In the case of one patient with $S$ pneumoniae infection a Sentinel bottle did not flag a positive, although the Bactec bottles did. However, when the growth curve of that Sentinel bottle was examined, it showed evidence of growth in the period before 12.5 hours. It is presumed that the organisms grew before and during this period and had become non-viable before the 12.5 hour minimum time for a positive result had elapsed.

There was a slightly higher false postive rate with Sentinel than with Bactec, probably due to operator error in incorrectly venting the Sentinel aerobic bottle.

It was difficult to determine accurately the time to positivity as this depended on the time of collection of the blood sample being recorded. Moreover, because the algorithm was deliberately configured so that it was not possible for the Sentinel to detect positive results during the first 12.5 hours, the Bactec had an inherent advantage during this period. The Bactec was indeed faster in detecting positive results up to the first 12 hours of the detection period. Since completion of the evaluation, the manufacturers of the Sentinel system have refined the algorithm to allow positive results to be detected at a minimum of $2 \cdot 25$ hours.

The Sentinel system had slightly fewer con-

Table 2 Time to detection of clinically important isolates for each system

\begin{tabular}{|c|c|c|c|c|c|c|c|c|c|}
\hline \multicolumn{10}{|l|}{ Time } \\
\hline $\begin{array}{l}\text { Days } \\
\text { Hours }\end{array}$ & $0-6$ & $6-12$ & $\begin{array}{l}1 \\
12-24\end{array}$ & $24-48$ & $\begin{array}{l}3 \\
48-72\end{array}$ & $\begin{array}{l}4 \\
72-96\end{array}$ & $\begin{array}{l}5 \\
96-120\end{array}$ & ${ }_{120-168}^{7}$ & Total \\
\hline $\begin{array}{l}\text { Bactec: } \\
\text { Number of strains isolated } \\
\text { Percentage cumulative positive }\end{array}$ & $\begin{array}{l}44 \\
20\end{array}$ & $\begin{array}{r}7 \\
23\end{array}$ & $\begin{array}{l}82 \\
61\end{array}$ & $\begin{array}{l}34 \\
77\end{array}$ & $\begin{array}{r}6 \\
79\end{array}$ & $\begin{array}{r}4 \\
81\end{array}$ & $\begin{array}{r}2 \\
82\end{array}$ & $\begin{array}{c}4 \\
84^{\star}\end{array}$ & 183 \\
\hline $\begin{array}{l}\text { Sentinel: } \\
\text { Number of strains isolated } \\
\text { Percentage cumulative positive }\end{array}$ & $\begin{array}{l}1 \\
0.5\end{array}$ & $\begin{array}{l}28 \\
13\end{array}$ & $\begin{array}{r}116 \\
67\end{array}$ & $\begin{array}{l}23 \\
77\end{array}$ & $\begin{array}{l}11 \\
82\end{array}$ & $\begin{array}{r}6 \\
85\end{array}$ & $\begin{array}{r}0 \\
85\end{array}$ & $\begin{array}{c}5 \\
87^{\star}\end{array}$ & 190 \\
\hline
\end{tabular}

${ }^{*}$ Bactec and Sentinel detected $84 \%$ and $87 \%$ of the total number of clinically important isolates. 
taminants than Bactec, perhaps representing the non-invasive monitoring technique of Sentinel.

When two blood culture systems are evaluated it is usual that a greater volume is taken than for one system and subsequently more isolations are made. The average volume of blood inoculated into both systems was $14.3 \mathrm{ml}$, less than the $20 \mathrm{ml}$ recommended. On average, a slightly higher volume of blood was introduced into the Sentinel bottles. Because the yield of organisms increases with the volume of blood introduced, ${ }^{8}$ it seems likely that both systems would have performed better if the recommended amount of blood had been introduced.

The continuous monitoring feature of Sentinel offers advantages in that the samples flagged as positive overnight are known immediately at the start of the day, enabling speedier reporting.

Continuous monitoring should also increase the speed of detection, although laboratory logisitics may need to be changed to make full use of this technology. For example, the best scenario would be for blood culture bottles to be entered into the system immediately on receipt in the laboratory, including during the night, rather than batching samples, as is commonly practised at present with other methods.

The only potential safety hazard was the use of a venting needle in the Sentinel aerobic bottle. Electricity was supplied to the instru- ment drawers at a maximum of 24 volts which was considered a good safety feature.

The Sentinel software is menu-based and controls the data collection and analysis function of the system; it also incorporates a data search facility for current and stored samples. The system was user friendly and was liked by staff involved with the evaluation.

The Sentinel system is a useful addition to the methods available for the detection of bacteria in blood.

This evaluation was supported financially by the Medical Devices Directorate, Department of Health

1 Shanson DC. Blood culture technique: current controversies. F Antimicrob Chemother 1990;25(Suppl C): 17-29.

2 Shanson DC. Modern blood culture techniques and other methods for detecting microbes in the blood. In: Shanson DC, ed. Septicaemia and endocarditis. Oxford, Oxford University Press, 1989:76-102.

3 Medical Devices Directorate. Difco Sentinel Blood Culture System. Report MDD/91/39. London: Department of System. Report

4 Corkhill JE, Rimmer K. Microbiological comparison of a new infra-red blood culture system (Bactec NR-660) and a radiometric system (Bactec 460). Med Lab Sci 1987;44:150-9.

5 Jungkind D, Millan J, Allen S, Dyke J, Hill E. Clinical comparison of a new automated infrared blood culture system with the BACTEC 460 system. $\mathcal{F}$ Clin Microbiol 1986;23:262-6.

6 Leitner C, ed. Geigy scientific tables. Basle: Ciba-Geigy 1984:67.

7 Ilstrup DM. Statistical methods employed in the study of blood culture media. In: Washington JA, ed. The detection blood culture media. In: Washington JA, ed. The de
of septicemia. Florida, USA: CRC Press, 1978:31.

8 of septicemia. Florida, USA: CRC Press, 1978:31. Controlled evaluation of the volume of blood cultured in Controlled evaluation of the volume of blood cultured in
detection of bacteraemia and fungaemia. $\mathcal{f}$ Clin Microbiol 1982;15:558-61. 